\title{
A FATAL CASE OF POISONING WITH CARBON TETRACHLORIDE
}

\author{
BY \\ H. J. R. KIRKPATRICK AND J. M. SUTHERLAND \\ From the Department of Pathology, the Royal Northern Infirmary, Inverness
}

(RECEIVED FOR PUBLICATION JULy 20, 1955)

Fatal cases of carbon tetrachloride poisoning are not encountered frequently in Great Britain. For this reason, and because it is felt that the histology obtained clearly demonstrates the toxic process, we report the following case.

\section{Case Report}

C. McI., a man aged 26 years, a joiner, on August 12. 1953, during the course of a bout of alcoholism. consumed a quantity of carbon tetrachloride in the form of "thawpit." Shortly afterwards he became intensely nauseated, but was unable to vomit for several hours. Thereafter, however, and for the following 48 hours vomiting was intractable. During this time he complained of pain in the chest, lower abdomen, and both lumbar regions, and experienced frequency of micturition.

On admission to a peripheral hospital on August 14 he did not look gravely ill, but a slight icteric staining of conjunctivae and skin was observed. He had a low-grade pyrexia $\left(99^{\circ} \mathrm{F}\right.$.) and there was evidence of mild bronchitis. The liver was slightly enlarged and tender on palpation. Examination of the urine disclosed a trace of albumin together with scanty red and white blood cells. Blood examination furnished the following results: haemoglobin. $13.3 \mathrm{~g}$. per $100 \mathrm{ml}$. : white blood cells, 12.400 per c.mm.

He was transferred to Raigmore Hospital, Inverness. on August 17. During the ensuing 24 hours the clinical picture did not alter materially, with the exception that there was evidence of patchy pulmonary consolidation, and the patient experienced occasional haemoptyses. Jaundice was slightly deeper but was never marked. The following investigations were carried out.

Urine analysis gave: albumin ++ , bilirubin ++ , red blood cells ++ , white blood cells + ; fine granular casts and epithelial debris were present.

The E.S.R. was $37 \mathrm{~mm}$. in one hour (Wintrobe). haemoglobin $13.8 \mathrm{~g}$. per $100 \mathrm{ml}$, and white blood cells 13.800 per c.mm. (polymorphs $76^{\circ}$. lymphocytes $22 \%$. monocytes $2 \%$ ).

No tubercle bacilli or other pathogens were isolated in sputum.

Blood cultures proved negative.
An agglutination reaction (including leptospira) was negative, as was a search for leptospira in the urine.

On August 19 a marked degree of oliguria developed followed within a few hours by symptoms and signs of a generalized haemorrhagic bronchopneumonia and pulmonary oedema. Gross cyanosis was associated with a respiratory rate of 40 per minute; pulse rate 110 per minute, temperature 100 F. Oliguria persisted and the patient died on August 20, eight days after the ingestion of carbon tetrachloricie.

The patient did not admit to exposure to any toxic material in his occupation. and it was only some time after his death that we learned he had consumed carbon tetrachloride. The history given above was reconstructed for the sake of clarity.

$\mathrm{He}$ was indeed suspected initially of suffering from leptospiral jaundice on the clinical evidence of hepatorenal injury, bronchitis. pyrexia. leucocytosis, and a history of occupational exposure to rats. This gives point to the contention of Partenheimer and Citron (1952) that carbon tetrachloride poisoning is often not diagnosed because it is not considered in the differential diagnosis and therefore a history of exposure is not sought.

\section{Necropsy}

Changes of pathological significance were limited to the liver, kidneys, and lungs.

Liver.-The liver was a little reduced in size, and its cut surfaces presented a fine mottling with pale and dark areas.

Histological Examination.-There was zonal necrosis of centrilobular distribution (Fig. 1). The changes were present in all lobules, though in variable degree, affecting from one-third to twothirds of the central zones in different lobules. In the necrotic areas there was infiltration with macrophages, phagocytosis of haemosiderin, and some retention of bile. The portal tracts showed a littie round-cell infiltration. In the peripheral zones of lobules the parenchymatous cells showed cloudy swelling and fatty infiltration. There was evidence 
of early regeneration of liver cells (Fig. 2). In the outer parts of the necrotic zones, single liver cells and groups of a few cells were present. There was nuclear hyperchromatism, an occasional cell was binucleate, and an occasional nucleus was in mitosis. In sections stained for reticulin fibres, the reticulin framework of the lobules was intact (Fig. 3).

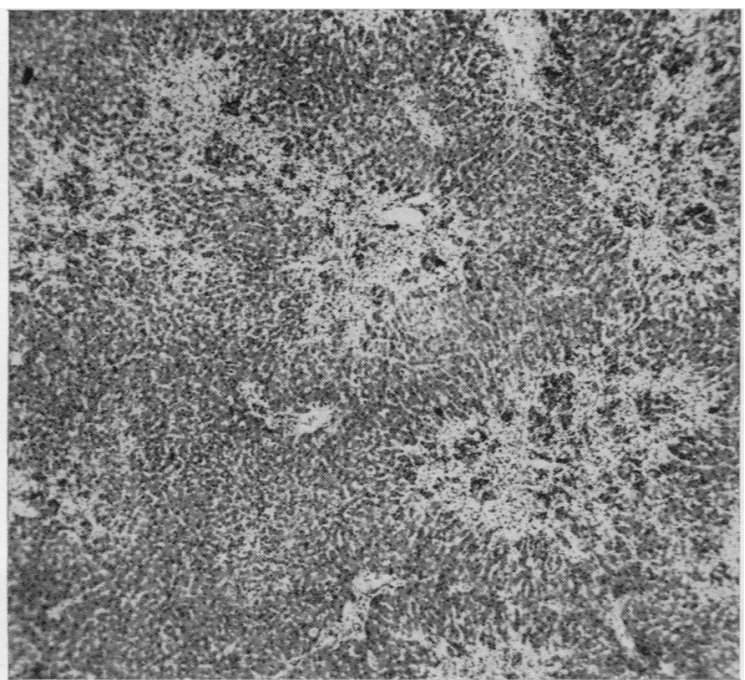

FIG. 1.-Section of liver showing centrilobular zonal necrosis $(\times 35)$.

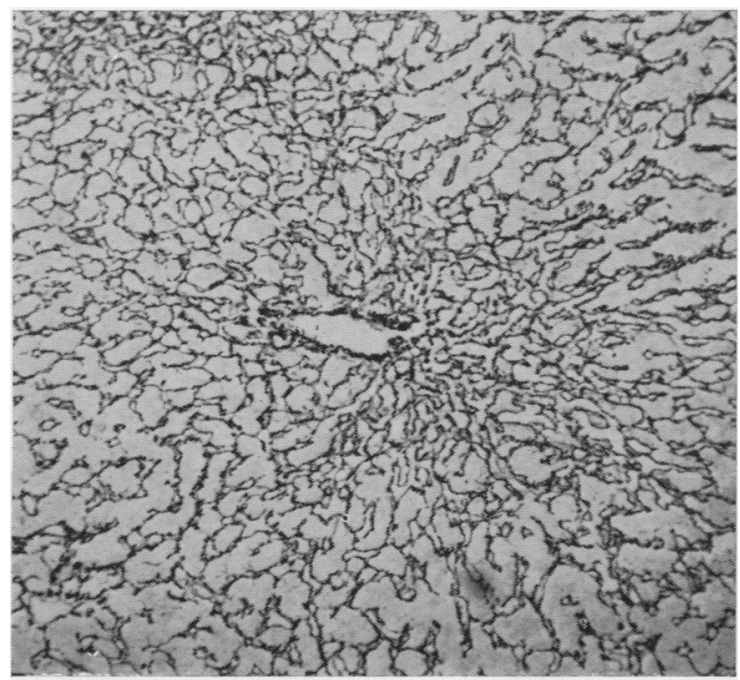

FIG. 3-Section of liver showing preservation of reticulin framework of liver lobule. (Foot's silver impregnation method fo- reticular fibres) $(\times 80)$.
Kidneys. - The kidneys were of normal size and congested, the congestion being more marked in the medulla than in the cortex.

Histological Examination.-The concentration of erythrocytes in capillary blood vessels indicated haemoconcentration and impairment of the renal circulation (Figs. 4, 5, and 8). Pathological changes were present in the glomeruli, tubules, and inter-

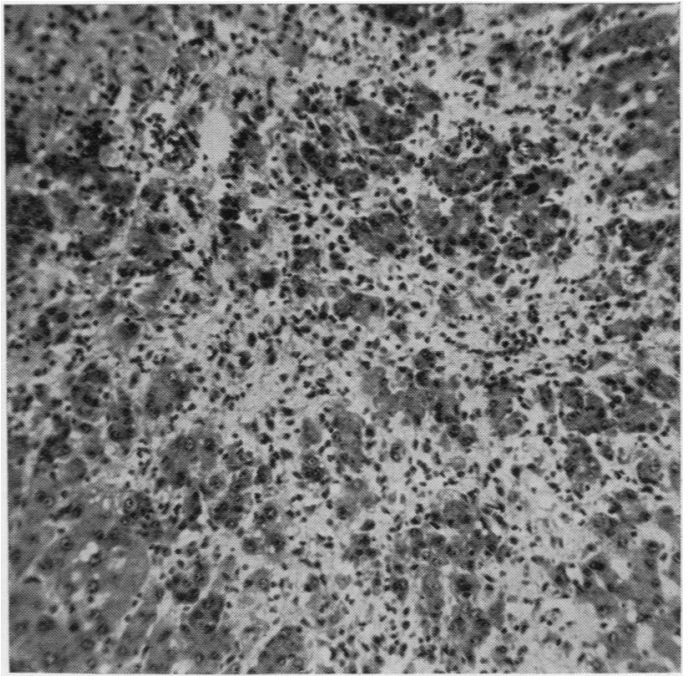

FIG. 2.-Section of liver showing inflammatory infiltration of necrotic zone and early regeneration of liver cells. $(\times 150)$.

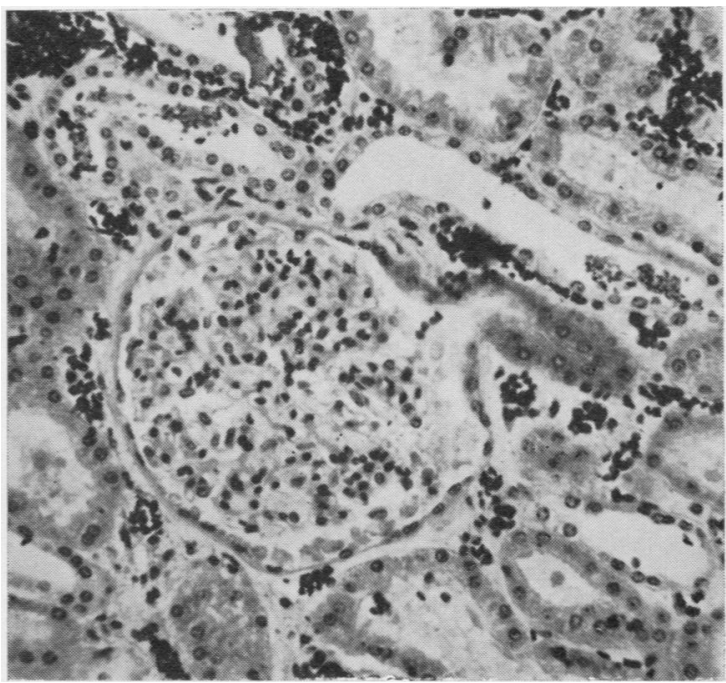

FIG. 4.-Section of kidney. A glomerulus showing swelling of tuft and degeneration and desquamation of capsular epithelium. Marked concentration of e:ythrocytes in tubular capillaries $(\times 200)$. 
stitial tissue. In the glomeruli the tufts were swollen, and there was degeneration and desquamation of the parietal and visceral epithelium of the capsules (Fig. 4). The cortical tubules were dilated, and in many tubules spurs projected into the lumen at points of angulation (Fig. 5). The epithelium of the proximal and distal parts of the nephrons was degenerated, but this was more

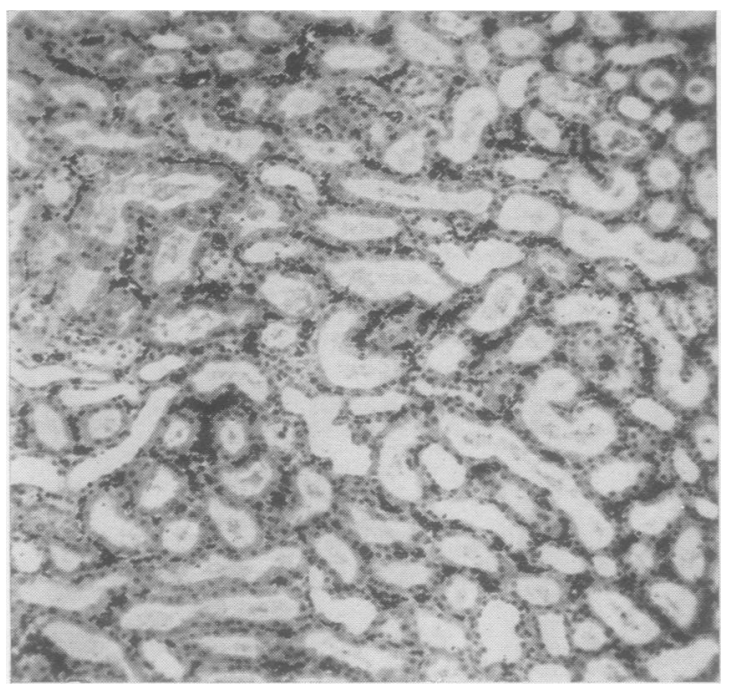

FIG. 5.-Section of kidney. Dilatation of cortical tubules, and spurs projecting into tubules at points of angulation. Epithelial debris in many tubules. Marked concentration of erythrocytes in tubular capillaries $(\cdot 80)$.

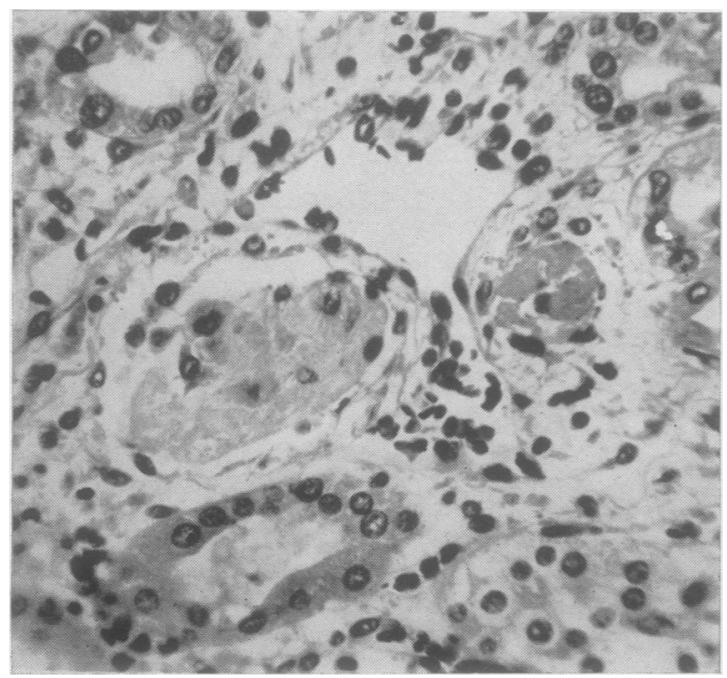

FIG. 7.- Section of kidney. In centre, two tubules occupied by remains of tubular epithelium and hyaline material. Below and above, tubules lined with regenerating epithelium $(x 350)$. marked in the distal parts. In these segments of the nephrons the lining epithelium was degenerated and necrotic. Many of the tubules contained epithelial debris, hyaline casts, and granular pigment material (Figs. 5, 6, 7, and 8). The pigment material was probably composed of altered erythrocytes derived from capillary haemorrhages into the lumen of the tubules (Fig. 8). In many

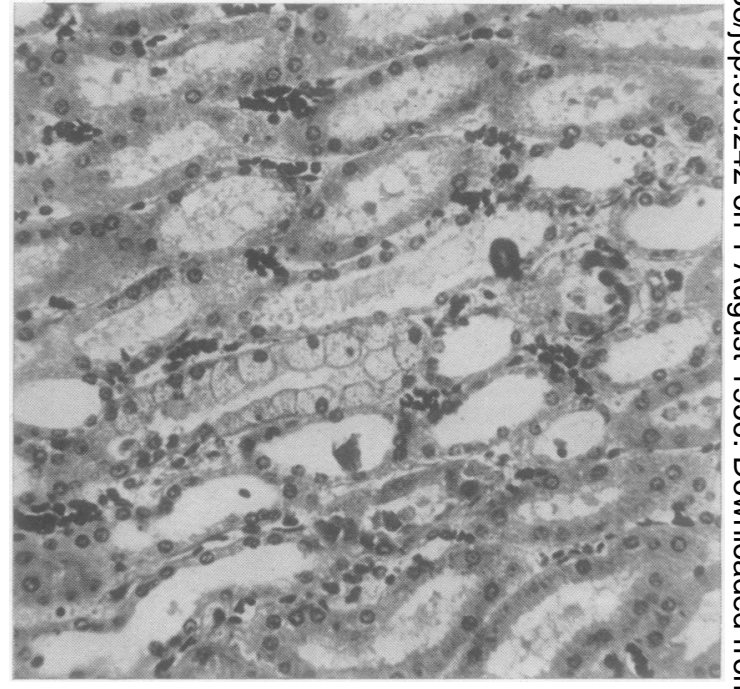

FIC. 6.-Section of kidney. In centre, a segment of Henle's loop lined by much swollen epithelium. Above this a similar segment occupied by debris from the disintegrated inner zone of lining epithelium. Nuclear zone largely intact and probably capable of regeneration $(\because 200)$.

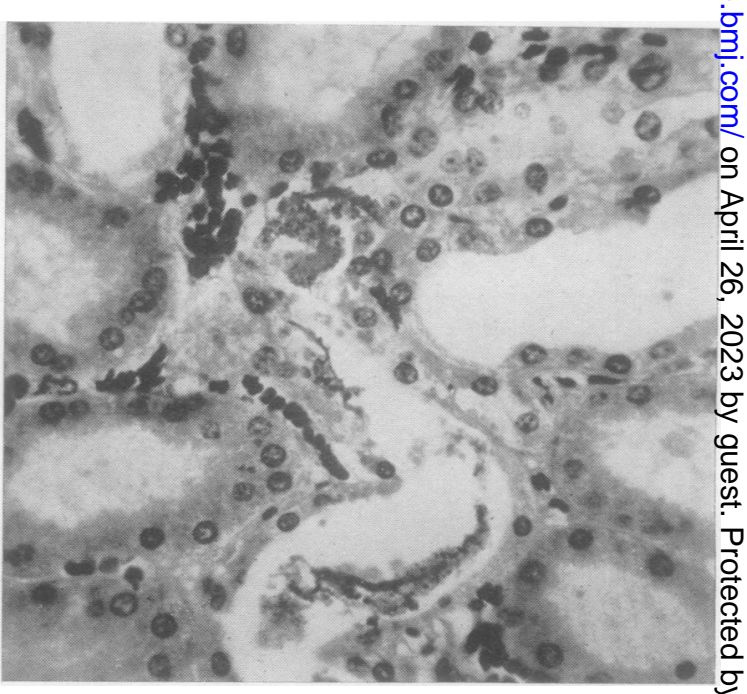

FIG. 8.-Section of kidney. In centre, segment of tubule containing granular pigment material. Note the apparent rupture of a $\bigcirc$ capillary into the upper end of the tubule $(x 350)$ 
tubules there was evidence of regeneration of the tubular epithelium. Segments of tubules were lined in part by cells with hyperchromatic nuclei of variable size, and an occasional nucleus was in mitosis (Figs. 7 and 9). Some tubules were filled with actively proliferating epithelial cells (Fig. 9). Interstitial changes were most marked in the junctional zone between cortex and medulla. In this

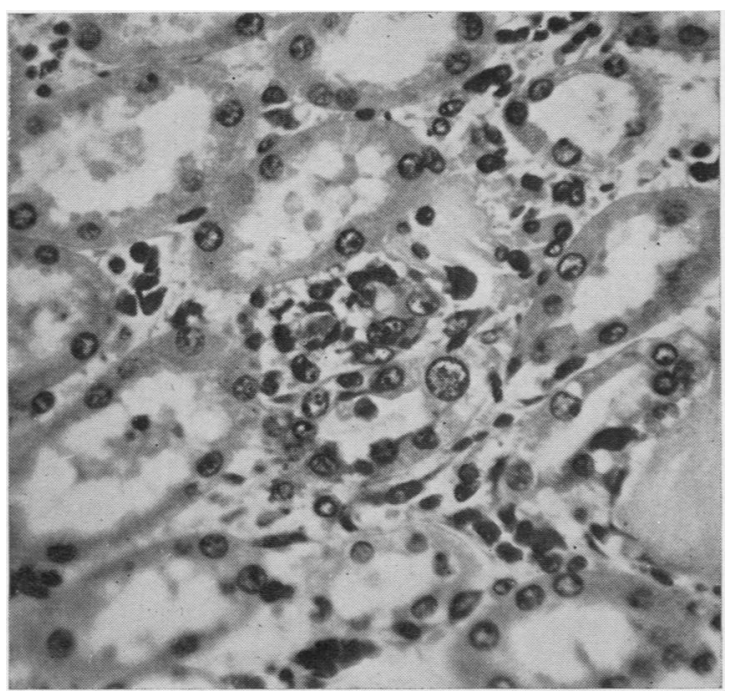

FIG. 9. - Section of kidney. In centre (a) a tubule lined by regenerating epithelium (note variable size of nuclei and nuclear hyperchromatism); (b) above this, a mass of proliferating epithelium in the lumen of a tubule $(\times 350)$.

zone there was inflammatory infiltration, chiefly with round-cells and a few eosinophils, which was maximal in relation to areas where tubules had broken down (Fig. 10).

Lungs.-The lungs were congested and oedematous.

Histological Examination.-There was congestion of capillaries and oedema, with haemorrhage into the alveoli and interstitial tissues in some areas. In many parts there was infiltration with macrophages, and in some areas phagocytosis of haemosiderin.

\section{Discussion}

Poisoning with carbon tetrachloride occasions (1) immediate effects, (2) delayed manifestations, and, in favourable cases, (3) a stage of recovery.

Immediate Effects. - The earliest symptoms of poisoning in this case were referable to the alimentary tract. This is in accordance with the experience of others, e.g., of Partenheimer and Citron (1952) and of Alha (1950), who note that the immediate state of poisoning may simulate acute abdominal emergencies.
Sirota (1949) and Partenheimer and Citron (1952) both report oliguria during this stage and ascribe it to dehydration. In the present case frequency of micturition was encountered due possibly to renal irritation, but we have no knowledge of the volume of urine passed.

The effect of the contemporaneous use of alcohol in enhancing the toxicity of carbon tetrachloride

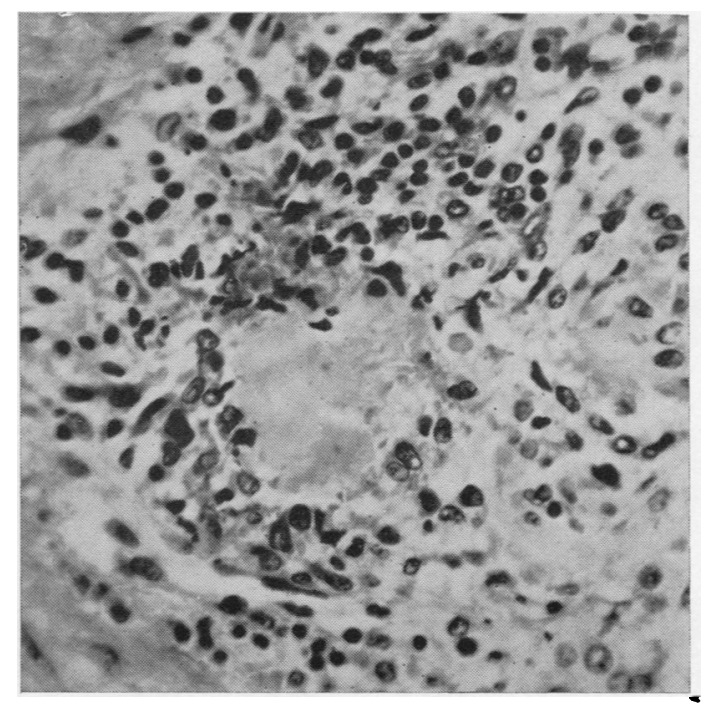

FIG. 10.-Section of kidney from junctional zone between cortex and medulla. Inflammatory infiltration related to broken-down tubule $(\times 350)$.

has been described by several workers including Moon (1950), Alha (1950), and Allebach and McPhee (1953). Moon suggests that alcohol and carbon tetrachloride may have a synergistic nephrotoxic as well as hepatotoxic effect, whereas Allebach and McPhee implicate the action of alcohol in increasing the solubility of carbon tetrachloride in biological fluids. Our patient, although not a chronic alcoholic, was drinking excessively when he ingested carbon tetrachloride. A companion was also induced to add "thawpit" to an alcoholic drink; the fact that he vomited immediately afterwards probably saved his life.

Delayed Manifestations. - Apart from occasional instances (Love and Miller, 1951), most fatal cases survive for a week or more after the initial exposure. The delayed manifestations occur as a rule one to three days after contact with the poison and they do not appear to be influenced by the route of administration. The organs principally affected are the liver, the kidneys, and the lungs. The clinical variations are manifold. In some cases liver damage predominates; in others the nephro- 
toxic effect is the arresting feature; in all pulmonary symptoms of greater or less degree probably occur.

The Liver.-Histological examination of the liver in this case demonstrated the centrilobular zonal necrosis characteristic of carbon tetrachloride poisoning. Himsworth (1947) has shown that the initial lesion is not necrosis but rather swelling of the parenchymal cells. This, in turn, impedes the intralobular circulation so that the blood supply to the parenchyma most distant from the inflow of blood is curtailed with resulting necrosis of these cells. Andrews and Maegraith (1948) have described these changes in rats and other animals following exposure to carbon tetrachloride.

In the human subject, Moon (1950) has shown that the severity of the hepatic lesion diminishes with longer periods of survival, and from the work of Umiker and Pearce (1953) it emerges that severe hepatic damage generally results in death during the first week. Initial injury of lesser severity is compatible with adequate recovery of hepatic function, due presumably to the capacity of the liver cells for rapid regeneration. In this case there is evidence of early regeneration of hepatic parenchymal cells eight days after the initial injury, and although there is widespread hepatic damage it is probable that death did not result from failure of liver function.

The Kidneys. - In the past emphasis has been placed on liver damage, but in recent years attention has been directed to the importance of the renal pathology. Smetana (1939) has shown that acute tubular necrosis occurs in carbon tetrachloride poisoning. Woods (1946) emphasized the similarity of the renal pathology in this condition to the renal pathology observed in the "crush syndrome." Moon (1950) found that morphological renal changes became progressively more pronounced with longer periods of survival, and that an inverse relationship existed between the severity of the hepatic and renal lesions. Whereas hepatic failure is the usual cause of death during the first week, Umiker and Pearce (1953) consider that renal insufficiency with uraemia is the important feature in patients who die after this time.

We are indebted to the studies of Sirota (1949) for a clearer understanding of the mechanism of renal failure in this condition. Within two to eight days after exposure oliguria develops, and is explained by back diffusion of the glomerular filtrate across damaged tubule walls. The presence of considerable protein and blood in the scanty urine reflects the existence of an abnormal glomerular permeability. In favourable instances this may be the only evidence of renal injury, but in more severe cases the phase of established oliguria or anuria, which may persist for periods up to three weeks, is reached. During this stage there is a marked diminution in renal blood flow and the resulting anoxia added to the initial toxic effect produces maximum depression of tubular function. The reduction in the renal blood flow is probably produced mechanically by increased intrarenal tension (Bull and Dible, 1953).

In favourable cases diuresis initiates the recovery process and probably represents re-establishment of the tubular barrier to the indiscriminate back diffusion of the filtrate. Although it is some time before selective reabsorption of the filtrate becomes established, the report of Simon (1939) indicates that the renal pathology can be completely resolved.

In the case reported here the predominant histological changes in the kidneys were those of lower nephron nephrosis, with impairment of the renal circulation and haemoconcentration. The evidence of active epithelial regeneration seen in many of the affected nephrons suggests that the kidneys had actually entered upon the recovery phase of the lesion. But the recovery of adequate renal function in such cases must depend on the rate and degree of re-establishment of continuity between proximal and distal segments of disorganized nephrons: on the re-estab!ishment of an adequate blood supply; and on the establishment of functional integrity of the regenerating renal epithelium. These changes were not sufticiently advanced to prevent the consequence of renal failure. Death occurred early in the phase of established oliguria, and was due to the retention of electrolytes and water, causing cardiac failure and death from pulmonary oedema.

The Lungs.-Alha (1950) notes the invariable occurrence of bronchitis. and Moon (1950) found pulmonary oedema to be present consistently and in greater degree in those who survived for longer periods. Umiker and Pearce (1953) found similar pulmonary lesions in 18 of 20 patients who lived longer than eight days after the fatal exposure. These changes occurred whether poisoning was by inhalation or ingestion, and a similar condition was found to obtain in miscellaneous uraemic states.

It would appear, therefore, that the pulmonary symptoms and the pathological changes which occasion them are entirely non-specific, and our findings are in accordance with this view. The occurrence of pulmonary oedema is related to the degree of renal damage. and Friedberg (1950) 
stresses the fact that the deficient excretion of sodium and water can produce the complete syndrome of congestive cardiac failure in the absence of cardiac disease. Similarly, Partenheimer and Citron (1952) ascribe the high mortality during the anuric stage to pulmonary oedema and hyperkalaemia.

Since modern techniques permit of accurate electrolyte balance and adequate nutrition during acute anuric episodes it is probable that this high mortality rate will be reduced. This, however, can only be achieved if the possibility of carbon tetrachloride poisoning is considered in cases presenting with a hepato-renal syndrome, and if the biochemical disturbance resulting from acute tubular necrosis is appreciated.

\section{Summary}

A case of poisoning by carbon tetrachloride is reported.

The histological findings in liver and kidneys are described.
The mechanism of the toxic process is discussed with reference to the morphological changes.

We are indebted to Dr. R. Ronald for permission to record the clinical data of this case, and to Mr. W. N. Rose for the preparation of the photomicrographs used to illustrate the pathology.

\section{REFERENCES}

Alha, A. (1950). Ann. med. intern. Fenn., 39, Suppl. 8 (English

Allebach, H. K. B., and McPhee, W. R. (1953). Missouri Med., 50,

Andrews, W. H. H., and Maegraith, B. G. (1948). Ann. trop. Med. Parasit., 42, 95.

Bull, G. M., and Dible, J. H. (1953). In Recent Advances in Pathology, 6th ed., ed. G. Hadfield, p. 281. Churchill, London.

Friedberg, C. K. (1950). Amer. J. Med., 9, 164 .

Himsworth, H. P. (1947). Lectures on the Liver and its Diseases, p. 32. Blackwell, Oxford.

Love, E. B., and Miller, A. A. (1951). Lancet, 1, 1306.

Moon, H. D. (1950). Amer. J. Path., 26, 1041

Partenheimer, R. C., and Citron, D. S. (1952). Arch. intern. Med., 89, 216.

Simon, M. A. (1939). Canad. med. Ass. J., 41, 580.

Sirota, J. H. (1949). J. clin. Invest., 28, 1412 .

Smetana, H. (1939). Arch. intern. Med., 63, 760.

Umiker, W., and Pearce, J. (1953). Arch. Path. (Chicago), 55, 203.

Woods, W. W. (1946). J. Path. Bact., 58, 767. 